\title{
História natural da síndrome de Kleine-Levin: revisão e discussão
}

\author{
Natural history of the Kleine-Levin syndrome: review and discussion
}

\author{
Dênio Lima'; J osé Antônio Zagalo-Cardoso" " \\ IPhD, Medicina, Instituto de Psiquiatria, University of London, Londres, Reino Unido. Professor \\ adjunto, Psiquiatria, Universidade de Brasília (UnB), Brasília, DF. \\ "Doutor, Psicologia da Saúde. Professor, Faculdade de Psicologia e de Ciências da Saúde \& Educação, \\ Universidade de Coimbra, Coimbra, Portugal. \\ Endereço para correspondência
}

\begin{abstract}
RESUMO
Neste artigo, os autores fazem um levantamento da história natural da síndrome de Kleine-Levin mediante a revisão e discussão críticas da literatura científica publicada até a data sobre o assunto. Prestam uma atenção especial aos correlatos psiquiátricos desta síndrome neuropsiquiátrica, focando os dilemas de diagnóstico deles decorrentes. Discutem as dificuldades e equacionam o perfil que os conhecimentos do estado da arte permitem delinear para a história natural da síndrome KleineLevin.
\end{abstract}

Descritores: Síndrome de Kleine-Levin, história natural, quadro clínico, curso evolutivo.

\begin{abstract}
In this article, we provide a picture of the so-called natural history of the Kline-Levin syndrome by means of a critical review and discussion of the scientific literature published so far about this topic. We focused special attention on the psychiatric correlates of this neuropsychiatric syndrome, addressing the diagnostic dilemmas raised from them. We also discuss the difficulties and show the profile that the knowledge about the state of the art in this area allows us to outline for the natural history of this syndrome.
\end{abstract}

Keywords: Kleine-Levin syndrome, natural history, clinical frame, clinical course.

\section{NTRODUÇÃO}

Objetivo

O presente artigo tem por objetivo delinear a história natural da síndrome de Kleine-Levin (SKL) a partir dos dados atualmente disponíveis com vistas a sistematizar o conhecimento da clínica dessa doença.

Em Medicina e em Psiquiatria, entende-se por história natural de uma afecção qualquer o seu quadro clínico, isto é, sua forma característica de apresentação e de evolução na ausência de 
intervenção dita iatrogénica, ou, em outras palavras, sem ser modificada pela iatria, etimologicamente, a arte ou processo de curar. A história natural seria, então, o conhecimento do padecimento, digamos assim, em sua forma espontânea ou pura. É a partir do conhecimento dela que se estabelecem as propedêutica (sintomatologia) e a patologia das afecções, bem como a história clínica com suas vertentes de diagnóstico e prognóstico.

A SKL é uma afecção relativamente rara que se inclui no grupo das hiperssonias idiopáticas intermitentes ou recorrentes e que, juntamente com a doença de Gélineau (narcolepsia-cataplexia), pertence ao grupo das hiperssonias patológicas primárias. A raridade da afecção impõe que o delineamento da sua história natural, diferentemente do que acontece com afecções comuns, só seja possível a partir de um levantamento histórico capaz de congregar a informação dispersa sobre ela na literatura científica.

\section{Breve levantamento histórico}

Duas descrições disputam a primazia quanto à menção de um quadro clínico correspondente à SKL: a do médico francês Edmé P. Chouvot de Beauchêne, de 1786, e a de Brierre de Boismont, de $1862^{1}$.

O psiquiatra alemão Willi Kleine, em 1925, na cidade de Frankfurt, foi o primeiro autor a descrever, de modo sistemático, um caso clínico com as características atribuídas à $\mathrm{SKL}$, em um artigo intitulado Periodische Schlafsucht ${ }^{2}$. Mais tarde, em 1929, o psiquiatra Max Levin confirmou as observações precedentes em um artigo dedicado à caracterização dos estados de sonolência mórbida ${ }^{3}$.

O epônimo "síndrome de Kleine-Levin" viria a ser criado e proposto em 1942 por Critchley \& Hoffman juntamente com a apresentação de dois casos clínicos pessoais ${ }^{4}$. Vinte anos mais tarde, Critchley $^{5}$, em artigo próprio, procedeu à apresentação de mais nove casos clínicos masculinos, perfazendo um total de 11 casos pessoais, e à revisão de outros 15 casos, também masculinos, a partir da literatura científica produzida até essa época. Nesse artigo clássico, o autor ponderou alguns aspectos básicos da síndrome, a saber: o predomínio de pacientes do sexo masculino; o padrão compulsivo e não propriamente bulímico da hiperfagia (isto é, um padrão "insaciável" e sem períodos de autoindução de vômito nem de jejum); e a tendência para a remissão espontânea ${ }^{5}$.

Como sinônimos para nomear a SKL são também usadas as designações "síndrome de hiperssonia e hiperfagia", "síndrome de hibernação", "síndrome familiar de hibernação", "sonolência periódica" e "fome patológica".

\section{MÉTODO}

Como vimos, o estudo da SKL carece de revisões sistemáticas e de meta-análises para elucidar muitos dos seus aspectos ainda ignorados ou discutidos, dentre eles sua história natural.

Para atingir nosso objetivo, a literatura consultada foi selecionada a partir de revistas internacionais relevantes de Psiquiatria e de Neurologia, em geral, e de perturbações do sono, em particular, privilegiando-se dois tipos de estudos, a saber: a) artigos de relatos de casos clínicos de SKL e b) artigos de grandes revisões da literatura científica sobre SKL. Para efetivar essa seleção, procedeu-se, em 3 de abril de 2007, a realizar uma pesquisa bibliográfica na base MEDLINE com base nas palavras-chave, em língua inglesa, Kleine-Levin syndrome (síndrome de Kleine-Levin), Kleine-Levin e periodic hipersomnia (hiperssonia periódica). A seleção foi feita, também, a partir das referências incluídas no arquivo eletrônico do jornal de neurologia Brain ${ }^{6}$ e no site da Fundação Kleine-Levin ${ }^{7}$. Dentre as dificuldades dessa seleção bibliográfica está a existência de artigos que apresentam alguns casos clínicos já publicados com anterioridade a eles e que se referem a casos de SKL sem critérios de diagnóstico fiáveis ou atribuíveis a outras afecções. O número de títulos encontrados parece ser elevado em face da raridade da SKL, traduzindo, talvez, a tendência compreensível a publicar cada caso que diagnosticado e, ultimamente, a vasta investigação 
neurobiológica, imunológica e genética que a síndrome suscita.

\section{DISCUSSÃO}

História natural e critérios de diagnóstico da SKL

A SKL tem um contorno neuropsiquiátrico, isto é, situado de modo limítrofe na fronteira entre a Neurologia e a Psiquiatria.

Do ponto de vista da Psiquiatria, é possível estabelecer analogias bastante curiosas com a SKL, dentre as quais se contam o caráter recorrente idêntico ao da chamada depressão endógena; a resposta terapêutica positiva de $41 \%$ dos pacientes aos estabilizadores do humor, particularmente ao lítio; a observação, em cerca da metade dos pacientes, de depressão durante os ataques e, em cerca de $8 \%$, de hipomania transitória durante a recuperação ${ }^{1}$. Verificam-se perturbações psiquiátricas como, por exemplo, alterações do caráter, comportamentos compulsivos (como nos casos raros descritos de cleptomania e de piromania) e/ou agitação ou amnésia (diminuição ou perda total da memória) dos eventos ocorridos durante o ataque em cerca de $50 \%$ dos casos ${ }^{1}$.

O diagnóstico da SKL é exclusivamente clínico, nele devendo preponderar a história médica e a história psicológica. O sexo masculino e a idade jovem do paciente são pontos de partida úteis para indiciar o problema, mas não são fatores suficientes para restringir a discussão das hipóteses de diagnóstico a esses agrupamentos.

Critchley apontou os principais critérios de diagnóstico da síndrome, a saber: hiperssonia periódica, hiperfagia, irritabilidade e disforia com hiperssexualidade, preponderância do sexo masculino e idade de início na juventude 5 .

No início dos anos 90, a Classificação Internacional de Doenças do Sono deixou de considerar obrigatórias tanto a hiperfagia quanto a perturbação do comportamento para definir a síndrome ${ }^{8}$.

Desde os trabalhos de Critchley, têm sido muito escassos os novos esclarecimentos dignos de nota sobre a síndrome apesar de a investigação ter sido conduzida em muitos sentidos, a saber: estudos poligráficos da estrutura do sono, padrões de secreção hormonal, anomalias do metabolismo dopaminérgico ou serotoninérgico, demonstração de um processo autoimunitário, identificação do tipo de antígenos do sistema leucocitário HLA ${ }^{1,9,10}$. É preciso sublinhar a inconsistência dos resultados obtidos em todas essas áreas, embora eles não constituam, propriamente, o escopo do nosso artigo.

A etiopatogênese da SKL não é conhecida ainda. Curiosamente, a apresentação clínica é sugestiva de uma relação fisiopatológica entre seus três sintomas principais que poderia muito bem resultar de disfunção hipotalâmica de caráter transitório. Na verdade, os centros psicofisiológicos relacionados ao sono, à fome e ao sexo têm uma localização de sede hipotalâmica e mantém uma relação neuroendócrina com a glândula pituitária (eixo hipotalâmico-hipofisário) através de seus conhecidos neuro-hormônios. Assim sendo, com base na clínica, seria legítimo admitir a existência de um deficit de saciedade do sono ou, por outras palavras, uma falta de satisfação com o sono: por mais que o paciente durma, não se sente repousado. No entanto, não tem sido possível, até agora, obter evidência de qualquer anomalia neuroendócrina definida e homogênea presente tanto durante os períodos sintomáticos (ataques) quanto em seus intervalos ${ }^{1,11}$.

Importância das revisões sistemáticas para a história natural da SKL

Em termos de história natural e clínicos, é importante ter presente os trabalhos de revisão de casuística anteriores como o de Orlosky ${ }^{12}$, com 30 pacientes; o de Gadoth et al. ${ }^{9}$, com 34 pacientes; o de Dauvilliers et al. ${ }^{11}$, com 30 pacientes; os de revisão de casos da literatura como o de Billiard \& Cadilhac $^{13}$, com 96 casos; e o mais recente de todos eles, com 186 casos $^{1}$. 
O primeiro simpósio científico dedicado à SKL em escala internacional teve lugar no âmbito da 20 ạ reunião anual da Sociedade do Sono, em 19 de junho de 2006, na cidade de Salt Lake City, EUA, e foi promovido pela Associated Professional Sleep Societies (APSS), que congrega duas associações da especialidade (a American Academic Sleep Medicine e a Sleep Research Society). Nesse evento, foram debatidos os conhecimentos atuais sobre a SKL.

Na Universidade de Stanford (Standford Center for Sleep Disorder), EUA, estabeleceu-se, em 2005, um programa de pesquisa científica intensivo visando a esclarecer as bases imunitárias, infecciosas, neurológicas e genéticas da SKL. Esse programa foi estabelecido sob a direção do Dr. Emmanuel Mignot e da Dra. I sabelle Arnulf e permitiu fazer uma vasta coleta de amostras de sangue de pacientes com SKL para pesquisas laboratoriais. Foi criado um questionário sistemático sobre os pacientes de SKL e seus familiares com vistas a organizar uma base informática de dados sobre a SKL. Esse grupo de trabalho começou por produzir uma extensa e exaustiva revisão de casuística a partir da literatura científica mundial, à qual dedicaremos, seguidamente, a nossa atenção ${ }^{1}$.

O projeto da Universidade de Stanford contribuiu para confirmar a noção de unidade da entidade clínica e de sua história natural pela verificação da semelhança do padrão de sintomas entre os pacientes e pela distribuição destes em escala mundial. Além disso, evidenciou como anomalias nucleares e permanentes da SKL a hiperssonia episódica (considerado o sintoma clínico "maior" e obrigatório para o diagnóstico, com a média diária de sono de $18 \pm 2$ horas, a mediana de 18 horas e o âmbito de variação entre as 12 e as 24 horas); e as perturbações cognitivas, registrando um maior grau de variabilidade nas perturbações do comportamento (alimentar e sexual) ${ }^{7,11-13}$.

Compilamos, aqui, alguns dos resultados dessa vasta revisão no sentido de caracterizar a história natural e o espectro sindromático da SKL. A grande maioria dos casos de SKL ( $98 \%$ ) foi de tipo esporádico, embora alguns casos fossem familiares (2\%). A proporção entre os sexos foi de $2: 1$ (masculino/feminino). Na maioria dos casos (61\%), ocorreu um fator precipitante representado, frequentemente, por uma infecção trivial (febre inespecífica, infecções respiratórias superiores, gastroenterites), mas também por outras ocorrências (abuso de substâncias como álcool ou marijuana, traumatismo craniano, privação de sono, esforço intelectual, sobrecarga emocional, menstruação, lactação, anestesia geral, exaustão física, etc.). O papel desses fatores precipitantes não é claro: em primeiro lugar, são muito heterogêneos e pouco específicos e, em segundo lugar, são muito mais raros antes dos episódios recorrentes de SKL (16\%). A média da idade de início da SKL foi de $16,9 \pm 8,5$ anos, a mediana foi de 15 anos e o âmbito de variação, entre os 4 e os 82 anos. Na verdade, $81 \%$ dos casos de SKL tiveram início durante a segunda década de vida. A duração clínica da SKL teve por mediana 8 anos, sendo mais prolongada nas mulheres e nos pacientes com menor frequência de episódios no primeiro ano. A média da idade de remissão da $\mathrm{SKL}$, para os 65 casos calculados, foi de $23 \pm 12$ anos. A mediana do número de episódios ou ataques, no decurso da doença e até a sua remissão, foi de sete (com uma média de 12 \$15 episódios e um âmbito de variação entre dois e 130 episódios). A mediana de duração dos episódios foi de 10 dias (com uma média de $12 \pm 9$ dias e um âmbito de variação entre 1 e 84 dias). A mediana do intervalo livre entre os episódios (ou período intercrítico) foi de 3,5 meses (com uma média de $6 \pm 10$ meses e um âmbito de variação entre 0,5 e 72 meses). Em 96,4\% dos casos, a descrição dos pacientes durante os períodos intercríticos não revelou qualquer alteração, embora em alguns casos os pacientes tenham referido um declínio acadêmico e uma disfunção mnésica moderada, mas prolongada. A possibilidade de disfunção residual ou sequelas após a remissão espontânea da SKL parece existir, mas não põe em causa o prognóstico benigno e, portanto, a recuperação completa, na generalidade dos casos estudados (160 em 168). Aliás, o compromisso cognitivo observado em menos de $4 \%$ dos casos pode não ser um efeito causado diretamente pela SKL, mas resultar de fatores indiretos como os efeitos colaterais sedativos da medicação ou a perda de aulas por faltas escolares. No âmbito do tratamento, foi possível verificar, com base nos relatos clínicos da literatura revista, o efeito terapêutico dos estimulantes anfetamínicos (12 em 17 pacientes) na sonolência e o do lítio (20 em 29 pacientes) na prevenção das recaídas ${ }^{1}$.

História natural e diagnóstico da SKL

Segundo é preconizado por Aldrich \& Naylor ${ }^{14}$, nas perturbações do sono, a história do sono deve ser detalhadamente elaborada perante uma resposta positiva às questões básicas de rastreio ("Tem dificuldade em iniciar o sono?" "Tem dificuldade em manter o sono?" "Tem excessiva sonolência 
diurna?" "Tem episódios perturbadores durante a noite?").

No estudo de alguns pacientes, pode haver indicação para recorrer a meios auxiliares de diagnóstico. Entre os instrumentos complementares de diagnóstico, podemos considerar os exames médicos (por exemplo, neurológico, bioquímicos) e fisiológicos (eletroencefalografia, polissonografia, imagiologia cerebral funcional por tomografia de emissão de positrões) e as escalas de autoavaliação mencionadas a seguir.

A Escala de Sonolência de Epworth, criada em Melbourne, Austrália, apresenta oito questões destinadas a avaliar o grau de sonolência diurna. As pontuações variam entre 0 e 24, considerando-se que resultados superiores a 16 indicam grave sonolência diurna. Assim, esta escala pode ajudar a excluir a fadiga muscular como causa de hiperssonia ${ }^{15}$, e, por outro lado, o questionário de dormir e despertar pode ser um instrumento útil para a avaliação clínica por ser breve, fácil e eficiente na detecção de perturbações do sono ${ }^{16}$.

Como mencionamos anteriormente, no âmbito do projeto de pesquisa sobre a SKL da Universidade de Stanford foi criado um questionário sistemático sobre os pacientes e seus familiares (Stanford Kleine-Levin Syndrome Questionnaire - R9) destinado à investigação. As suas características são mais adequadas para a pesquisa do que, propriamente, para o uso clínico ${ }^{17}$.

Os testes formais de cognição e memórias podem ser de aplicação difícil e levar a resultados comprometidos quando os pacientes se encontram em estado de pouca cooperação, irritabilidade, sonolência ou falta de atenção.

Os exames médicos não costumam revelar qualquer alteração, embora isso possa ser importante para garantir, nomeadamente, a ausência de sinais neurológicos sugestivos de lesão focal ou meningite. Na verdade, com exceção das síndromes de apneia do sono, os exames físicos das hiperssonias não são, habitualmente, reveladores do problema. No entanto, convém frisar que os exames complementares podem ser de interesse para corroborar o diagnóstico diferencial, nomeadamente no sentido de excluir possíveis causas da sintomatologia como a epilepsia (EEG), lesões focais cerebrais (imagiologia do sistema nervoso central) e meningite ou encefalite (análise do líquido cefalorraquidiano).

O registro poligráfico pode revelar uma estrutura normal do sono, acompanhada de decréscimo de eficiência e de despertares frequentes durante o estádio II do sono ${ }^{11}$. Durante os episódios de hiperssonia, o registro poligráfico de 24 ou 48 horas pode contribuir para o diagnóstico com algumas informações como as relativas ao sono fragmentado e instável, à redução nos estágios III e IV do sono sem movimentos oculares rápidos (MOR) e à redução do tempo de latência do sono $\mathrm{MOR}^{18}$.

História natural e erros de diagnóstico da SKL

A literatura reporta, frequentemente, erros de diagnóstico na SKL aos quais aludiremos seguidamente, sendo para melhor evitar esses erros que o conhecimento da história natural da síndrome se afigura indispensável.

No que se refere à SKL, a literatura científica pode estar facilmente distorcida, por relatar, quase que exclusivamente, os casos mais graves e mais homogêneos da síndrome ${ }^{1}$ e, assim, enviesar a noção clínica sobre a história natural da SKL e, portanto, sobre as formas de apresentação e o curso da afecção.

Alguns autores consideram que os casos de síndrome com apresentação incompleta são mais numerosos do que os casos de apresentação completa ${ }^{12,13,19}$.

História natural e diagnóstico diferencial da SKL

A SKL é uma entidade clínica que se localiza na fronteira entre a Neurologia e a Psiquiatria, sendo essa uma dificuldade para a interpretação de suas manifestações. É possível que seja uma afecção 
neurológica com sintomatologia psiquiátrica ${ }^{8}$, mas isso não constitui sequer uma certeza.

Como se depreende do exposto acima, é extremamente limitativo o fato de contar com um diagnóstico exclusivamente clínico ${ }^{19}$ e a falta de um sintoma patognomônico na história natural da SKL que, por si só, permita defini-la. Além disso, os intervalos intercríticos da SKL são completamente assintomáticos, escapando a qualquer exploração clínica ou método complementar de diagnóstico. Apenas os períodos paroxísticos (ataques, crises ou episódios) podem contribuir para um esclarecimento ${ }^{18}$.

Parece haver um grande polimorfismo da sintomatologia da SKL, nomeadamente ao nível da riqueza e da diversidade da própria sintomatologia psiquiátrica ${ }^{20}$.

Outro aspecto que limita o diagnóstico de SKL é a ausência de um marcador biológico (orgânico, laboratorial, analítico, genético) típico da afecção. Isso acaba por dificultar, também, a clarificação da existência de uma entidade clínica realmente uniforme.

Por fim, vejamos os diagnósticos diferenciais com que nos deparamos à luz da história natural da SKL no âmbito das perturbações do sono, perturbação do biorritmo (ritmo circadiano sono-vigília), perturbações médicas, perturbações por abuso de substâncias, perturbações mentais (psiquiátricas).

\section{Perturbações do sono}

Existem cerca de sete dezenas de perturbações do sono, das quais quase todas revestem a forma crônica, causando ao paciente uma significativa sobrecarga emocional e interferindo com o seu funcionamento social ou ocupacional. É habitual classificar as perturbações do sono, de acordo com a sua causa, em primárias (causadas por perturbações mentais e doenças orgânicas) e secundárias (causadas por prescrição de medicamentos e abuso de substâncias).

As duas grandes categorias de perturbações primárias do sono são as dissonias e as parassonias. A mais importante das dissonias é a insônia primária, que começa, habitualmente, no jovem adulto ou na idade média da vida, podendo ter por efeito secundário períodos diurnos de hiperssonia de caráter compensatório. Não sendo, propriamente, uma perturbação do sono em si mesma, o sono insuficiente ou a privação do sono são geradores de sonolência diurna.

Dentro das perturbações do sono, as outras perturbações intermitentes ou recorrentes do sono constituem um importante problema de diagnóstico diferencial. Dessas hiperssonias, mencionamos a síndrome dos fusos horários, que pode, nos casos de maior frequência de voos a cruzar fusos horários, revestir um caráter crônico e, por isso mesmo, assemelhar-se à SKL; e a hiperssonia associada à menstruação. Esta forma de hiperssonia deve ser ponderada atentamente ${ }^{21}$.

A apneia obstrutiva do sono (síndrome de hipopneia) e a SKL podem, eventualmente, confundir-se entre si. Como a apneia obstrutiva do sono é tratada, habitualmente, por pneumologistas, isso implica que esses especialistas devem estar, também, sensibilizados para essa possibilidade de confusão entre as duas afecções.

Há perturbações do sono que não comprometem a duração do sono, mas que podem apresentar um ou outro dos sintomas da SKL como é o caso das alucinações hipnagógicas e hipnopômpicas. Este aspecto torna-se relevante na medida em que as perturbações do sono podem ser comórbidas, isto é, simultâneas em um mesmo paciente.

\section{Perturbação do biorritmo (ritmo circadiano sono-vigília)}

Há várias causas, tanto endógenas quanto ambientais, para a desregulação do relógio biológico do núcleo supraquiasmático do hipotálamo, que regula o ritmo de sono-vigília. O trabalho por turnos pode ser muito disruptivo para os ritmos naturais de sono-vigília e originar hiperssonia diurna. 0 mesmo acontece com a síndrome dos fusos horários acima referida. 
Atendendo à letargia (falta de energia) que caracteriza a SKL, o primeiro problema a partir do qual se deve fazer o diagnóstico diferencial é o da fadiga muscular, idéntico ao que se verifica na fibromialgia. A síndrome de fadiga crônica e a SKL podem confundir-se entre si e carecer de diagnóstico diferencial ${ }^{22}$.

Entre as afecções médicas que levantam o problema do diagnóstico diferencial em relação à SKL, contam-se a anemia, a encefalite viral e outras doenças orgânicas do cérebro (traumatismo, tumor), as doenças metabólicas (hipotiroidismo, uremia, hipoglicemia, hipercalcemia, hipercapnia, insuficiência hepática). Afecções como a esclerose múltipla ou a obesidade podem gerar quadros de hiperssonia, tal como certas infecções de que são exemplo o vírus de Epstein-Barr e herpes-zóster ou a mononucleose infecciosa. No foro neurológico, merecem especial menção a epilepsia e as disfunções do sistema nervoso autônomo. A síndrome de Klüver-Bucy é outra afecção rara caracterizada por hiperfagia, hiperssexualidade e compromisso emocional. É necessário ter presente a existência de casos de início tardio ( $>35$ anos) e mesmo geriátricos, que podem ocorrer após os $80 \operatorname{anos}^{23}$. Nas faixas etárias mais avançadas, também merecem menção a doença de Parkinson e a doença de Huntington.

\section{Perturbações por abuso de substâncias}

A drogadição, tanto por drogas ilícitas quanto por psicofármacos, constitui um possível diagnóstico diferencial a ter em conta na SKL. Por exemplo, o álcool e a cafeína podem originar, secundariamente, hiperssonia diurna. Os opiáceos tornam os consumidores recentes bastante sonolentos. A hiperssonia é típica da síndrome de abstinência de substâncias como as anfetaminas ou a cocaína. Fármacos como o triazolam provocam uma síndrome idêntica à SKL.

\section{Perturbações mentais (psiquiátricas)}

A frequência e a diversidade de sintomas do foro mental que podem manifestar-se na SKL impõem, por si mesmas, uma atenção e um cuidado clínicos especiais no âmbito do sempre necessário diagnóstico diferencial da síndrome em relação às perturbações mentais. Com efeito, o complexo sindromático de perturbações cognitivas associadas a perturbações do sono e do apetite constituem a base para o diagnóstico de certas perturbações mentais. Além disso, o predomínio das perturbações cognitivas e comportamentais pode obscurecer o diagnóstico da $\mathrm{SKL}^{20}$.

Segundo o estudo da revisão sistemática de Arnulf et al. ${ }^{1}$, identificaram-se, durante os episódios de SKL, os sintomas que passamos a enumerar, por ordem decrescente de frequência, em cada um dos agrupamentos considerados. No âmbito das perturbações cognitivas (presentes em todos os pacientes em alguma de suas formas): transtornos do discurso (mutismo, ausência de discurso espontâneo, monossílabos ou vocabulário limitado, incoerente, linguagem estereotipada infantil, lentidão para falar ou compreender, perseverações ou ecolalia, nas respostas); confusão mental (desorientação); deficit de atenção, deficit de concentração, deficits de memória (amnésia relativa aos fatos ocorridos durante os ataques), desrealização, alucinações, delírios. No contexto das perturbações do comportamento alimentar (verificados em 3/4 dos pacientes em alguma de suas formas): hiperfagia (ingestão até três vezes superior à quantidade habitual de comida em seis a oito refeições por dia), apetite para doces, polidipsia, roubo de alimentos, falta de apetite, bizarrias do ato de comer como incapacidade de parar de comer. Perturbações do humor, presentes em metade dos pacientes: depressão durante os episódios, incluindo ideias ou mesmo tentativas de suicídio, e euforia, sob a forma de hipomania nos períodos intercríticos; embotamento afetivo; estados de ansiedade; irritabilidade, agitação. Perturbações compulsivas do comportamento, presentes em metade dos pacientes, incluindo: hiperssexualidade (masturbação em público ou muito frequente, linguagem obscena, manipulação e exibição dos órgãos genitais e tentativas indiscriminadas de abuso sexual); compulsões para cantar ou escrever ${ }^{1}$.

A relação entre perturbações do sono e perturbações psiquiátricas é muito estreita, sendo relativamente bem conhecida nos adultos (por exemplo, no caso do papel da insônia e da hiperssonia no desenvolvimento ou recorrência ulterior de síndromes depressivas, estados de ansiedade ou abuso de substâncias). Na infância e na adolescência, ainda precisa ser esclarecido até 
que ponto as perturbações do sono constituem fatores de vulnerabilidade para as perturbações mentais ${ }^{24}$. Em contrapartida, muitas perturbações mentais podem originar alterações do sono, sendo mesmo a causa mais frequente de insônia crônica.

Ao discutir os correlatos psiquiátricos da SKL temos que ponderar não apenas a relação entre a síndrome e as perturbações afetivas, mas também o possível impacto emocional dos ataques ${ }^{25}$. $\mathrm{Na}$ verdade, os ataques representam, por si mesmos e pelas suas implicações intelectuais, laborais, familiares e sociais, uma fonte de mal-estar, de compromisso da qualidade de vida e um potencial deflagrador de repercussões mentais. As perturbações do sono, em particular as mais persistentes, podem produzir efeitos prejudiciais nas funções psicológicas. O diagnóstico e o tratamento precoces, então, revestem-se de um considerável valor preventivo.

Quanto ao diagnóstico diferencial em relação à $\mathrm{SKL}$, é preciso ponderar, especialmente, a depressão, as perturbações de ansiedade, a histeria (perturbações de conversão), a bulímia nervosa, a esquizofrenia, no seu período inicial, o transtorno do humor bipolar e o surto psicótico (psicose aguda). A SKL pode constituir, em certos casos, um modo de início das psicoses funcionais. A SKL distingue-se da bulimia nervosa em que a perturbação do comportamento alimentar da primeira não é acompanhada de preocupação excessiva com a forma e o peso do corpo, nem da alternância com períodos de autoindução do vômito que caracterizam a segunda ${ }^{1}$.

\section{CONCLUSÃO}

A SKL é uma entidade clínica que se localiza na fronteira entre a Neurologia e a Psiquiatria, sendo essa uma dificuldade para a interpretação das manifestações de seu quadro clínico.

O desconhecimento da etiologia e da fisiopatologia da SKL não permitem sair da esfera estritamente clínica e acentuam a importância do esclarecimento de sua história natural. Em face de uma síndrome rara, é necessário lançar mão de levantamentos históricos, de revisões sistemáticas e de meta-análises com vistas a sistematizar esse conhecimento, tal como se procurou fazer neste artigo ao rever e discutir, criticamente, a história natural da síndrome no intuito de equacioná-la de um modo mais objetivo.

\section{REFERÊNCIAS}

1. Arnulf I, Zeitzer JM, File J, Farber N, Mignot E. Kleine-Levin syndrome: a systematic review of 186 cases in the literature. Brain. 2005; 128:2763-76.

2. Kleine W. Periodische Schlafsucht. Mschr Psychiat Neurol. 1925;57:285-320.

3. Levin M. Narcolepsy (Gelineau's syndrome) and other varieties of morbid somnolence. Arch Neurol Psych. 1929;22:1172-200.

4. Critchley M, Hoffman HL. The syndrome of periodic somnolence and morbid hunger (KleineLevin). Br Med J. 1942; 1:137-9.

5. Critchley M. Periodic hypersomnia and megaphagia in adolescent males. Brain. 1962;85:627-56.

6. Brain, a journal of neurology [website]. Oxford J ournals. http://brain.oxfordjournals.org . Acessado: dezembro de 2005.

7. Kleine-Levin Foundation Inc [website]. Nov 2007.

8. Diagnostic Classification Steering Committee (DCSC) (Thorpy MJ , chairman). International Classification of Sleep Disorders: Diagnostic and Coding Manual. Rochester: American Sleep Disorders Association; 1990.

9. Gadoth N, Kesler A, Vainstein G, Peled R, Lavie P. Clinical and polysomnographic characteristics 
of 34 patients with Kleine-Levin syndrome. J Sleep Res. 2001; 10:337-41.

10. Dauvilliers Y, Mayer G, Lencendreux M, Neidhart E, Peraita-Adrados R, Sonka K, et al. Kleine-Levin syndrome: an autoimmune hypothesis based on clinical and genetic analyses. Neurology. 2002;59:1739-45.

11. Mayer G, Leonhard E, Krieg J, Meier-Ewert K. Endocrinological and polysomnographic findings in Kleine-Levin syndrome: no evidence for hypothalamic and circadian dysfunction. Sleep. 1998;21:278-84.

12. Orlosky MJ . The Kleine-Levin syndrome: a review. Psychosomatics. 1982;23:609-21.

13. Billiard M, Cadilhac J. Lees hypersomnies récurrentes. Rev Neurol. 1988; 144:249-58.

14. Aldrich MS, Naylor MW. Approach to the patient with disordered sleep. In: Kryger MH, Roth T, Dement WC, eds. Principles and Practice of Sleep Medicine. Philadelphia: Saunders; 2000. p. 521-5.

15. Johns MW. A new method for measuring daytime sleepiness: the Epworth sleepiness scale. Sleep. 1991; 14.540-5.

16. Roth T, Zammit G, Kushida C, Doghamji K, Mathias SD, Wong JM, et al. A new questionnaire to detect sleep disorders. Sleep Med. 2002;3(2):99-108.

17. Kleine Levin Syndrome Questionnaire Stanford Center for Sleep. med.stanford.edu/school /psychiatry/klsquestionnaire.pdf . Acessado: fevereiro de 2007.

18. Minvielle S. Le syndrome de Kleine-Levin: une afection neurologic à symptomatologie psychiatrique. Encephale. 2000;26:71-4.

19. Sadeghi M. Kleine-Levin syndrome. A report of three adolescent female patients. Arch Iran Med. 1999;2:1-3.

20. Masi G, Favilla L, Millepiedi S. The Kleine-Levin syndrome as a neuropsychiatric disorder: a case report. Psychiatry. 2000;63:93-100.

21. Papy JJ, Conte-Devolx B, Sormani J, Porto R, Guillaume V. Syndrome d'hipersomnie periodique avec megaphagie chez une jeune femme, rythme par le cycle menstruel. Rev Electroencephalogr Neurophysiol Clin. 1982; 12:54-61.

22. Marcelli D. Enfance et psychopathologie. Collection Les âges de la vie. Paris: Masson; 1999.

23. Gau SF, Soong WT, Liu HM, How JW, Tsai WY, Chiu YN, et al. R. Kleine-Levin syndrome in a boy with Prader Willi syndrome. Sleep. 1996;19(1):13-7.

24. Stores G. Misdiagnosing sleep disordes as primary psychiatric conditions. Advan Psycht Treatment. 2003; 9:69-77.

25. Papacostas SS, Hadjivasilis V. The Kleine-Levin syndrome. Report of a case and review of the literature. Eur Psychiatry. 2000;15:231-5.

\section{ب̣̂ Correspondência}

Dênio Lima

SQN 316, Bloco F, apto 204

CEP 70775-060, Brasília, DF

E-mail: denio.lima@gmail.com

Recebido em 22/07/2007. 
Aceito em 12/09/2007.

Não foram declarados conflitos de interesse associados à publicação deste artigo. 